Preprint SSU-HEP-98/04

Samara State University

\title{
Evaluation of hadronic vacuum polarization contribution to muonium hyperfine splitting
}

\author{
R.N. Faustov \\ Scientific Council for Cybernetics, \\ Russian Academy of Sciences \\ 117333, Moscow, Vavilov, 40, Russia, \\ A. Karimkhodzhaev \\ Department of Physics, Tashkent State University, \\ 700095, Tashkent, Uzbekistan, \\ A.P. Martynenko \\ Department of Theoretical Physics, Samara State University, \\ 443011, Samara, Pavlov, 1, Russia
}

\begin{abstract}
The contribution of hadronic vacuum polarization (HVP) to the hyperfine splitting of the muonium ground state is evaluated with the account of modern experimental data on the cross section of $e^{+} e^{-} \rightarrow$ annihilation into hadrons.
\end{abstract}


In the framework of the quasipotential method the particle interaction operator for $1 \gamma$ - and $2 \gamma$ - processes takes the form [1, 2, 3]:

$$
\begin{gathered}
V=V_{1 \gamma}+V_{2 \gamma}=V^{c}+\Delta V, \\
V_{1 \gamma}=T_{1 \gamma}, \quad V_{2 \gamma}=T_{2 \gamma}-T_{1 \gamma} \times G^{f} \times T_{1 \gamma},
\end{gathered}
$$

where $V^{c}$ is Coulomb potential, $T_{1 \gamma}, T_{2 \gamma}$ are one- and two-photon scattering amplitudes off the energy shell, $\left[G^{f}\right]^{-1}=\left(b^{2}-\vec{p}^{2}\right) / 2 \mu_{R}$ is the free two-particle Green function $\left(\mu_{R}\right.$ is relativistic reduced mass) [4]. Then the corresponding correction in the energy spectrum may be written as follows:

$$
\Delta E=<\psi_{n}^{c}|\Delta V| \psi_{n}^{c}>
$$

where $\psi_{n}^{c}$ is the ordinary Coulomb wave function. The hadronic vacuum polarization contribution to the energy spectrum is determined by the Feynman diagrams represented on figure by means (1). To take into account vacuum polarization in these one-loop diagrams, we must do the following substitution in the photon propagator [2]

$$
\frac{1}{k^{2}+i \varepsilon} \rightarrow\left(\frac{\alpha}{\pi}\right)^{2} \int_{s_{t h}}^{\infty} \frac{\rho(s) d s}{k^{2}-s+i \varepsilon}
$$

where the spectral function $\rho(s)$ is connected with known cross section of $e^{+} e^{-}-$annihilation into hadrons $\sigma^{h}$ :

$$
\rho(s)=\frac{R(s)}{3 s}=\frac{\sigma^{h}\left(e^{+} e^{-} \rightarrow \text { hadrons }\right)}{3 s \sigma_{\mu \mu}\left(e^{+} e^{-} \rightarrow \mu^{+} \mu^{-}\right)},
$$

and $\sigma_{\mu \mu}\left(e^{+} e^{-} \rightarrow \mu^{+} \mu^{-}\right)=4 \pi \alpha^{2} / 3 s$ is the $e^{+} e^{-}$- annihilation cross section to muonic pair. Introducing Feynman's parameterization (parameter $\lambda$ ), we can write the contribution of HVP to muonium ground state hyperfine splitting in the form [2]:

$$
\Delta E_{h f s}^{h}(\mu e)=\left(\frac{\alpha}{\pi}\right)^{2} m_{e} m_{\mu} \tilde{E}_{F} \int_{4 m_{\pi}^{2}}^{\infty} \frac{R(s) d s}{3 s} \int_{0}^{1} \frac{16-6 \lambda-\lambda^{2}}{m_{\mu}^{2} \lambda^{2}+s(1-\lambda)} d \lambda
$$

$m_{e}, m_{\mu}, m_{\pi}$ are the masses of electron, muon and charged pion, $\tilde{E}^{F}=8 \alpha^{4} m_{e}^{2} m_{\mu}^{2} / 3\left(m_{e}+\right.$ $\left.m_{\mu}\right)^{3}$ is the Fermi energy of the muonium. The inner integral in (5) can be calculated analytically. For the calculation of external integral we have used the parameterization of $\mathrm{R}(\mathrm{s})$, which accounts the modern experimental data on annihilation cross section of $e^{+} e^{-}$ into hadrons [5, 6, 7, 8, 9, 10, 11, 12, 13].

The cross section $\sigma^{h}$ may be expressed as a sum of terms [2, 3]:

$$
\sigma^{h}=\sigma_{\pi \pi}^{h}+\sigma_{\text {res }}^{h}+\sigma_{\text {background }}^{h} .
$$

The main contribution to the cross section $\sigma^{h}$ is determined by the process $e^{+}+e^{-} \rightarrow$ $\pi^{+}+\pi^{-}$. The cross section of this reaction is proportional to the modulus squared of pion form factor $F_{\pi}$. The corresponding spectral function (4) may be presented in the form:

$$
\rho_{\pi \pi}(s)=\frac{\left(s-4 m_{\pi}^{2}\right)^{3 / 2}}{12 s^{5 / 2}}\left|F_{\pi}(s)\right|^{2}
$$



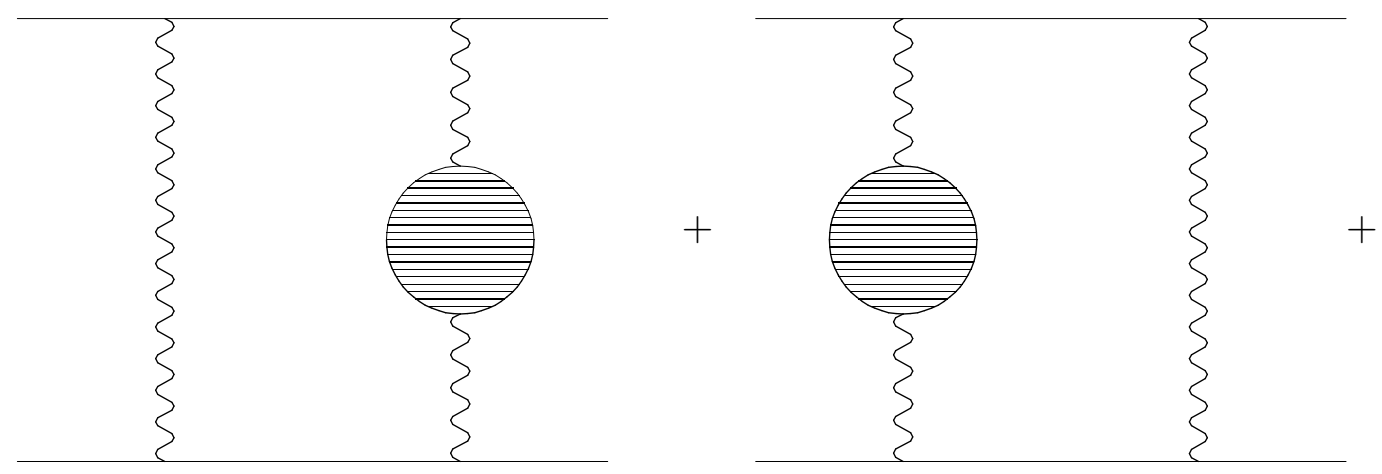

diagrams

with crossed

+ photon lines

and subtracted

diagrams

Figure 1: Feynman diagrams, defining the hadronic vacuum polarization contribution to hyperfine structure of atom $(\mu e)$

Contrary to our recent calculation [14 to perform integrations in (5) for this case we have used the expression of pion form factor, obtained in [15]:

$$
F_{\pi}(s)=\frac{m_{\rho}}{m_{\rho}^{2}-s-i m_{\rho} \Gamma_{\rho}(s)} \exp \left\{-\frac{s}{96 \pi^{2} f_{\pi}^{2}} \operatorname{Re}\left[A\left(\frac{m_{\pi}^{2}}{s}, \frac{m_{\pi}^{2}}{m_{\rho}^{2}}\right)+\frac{1}{2} A\left(\frac{m_{K}^{2}}{s}, \frac{m_{K}^{2}}{m_{\rho}^{2}}\right)\right]\right\}
$$

where the function

$$
\begin{gathered}
A\left(\frac{m_{P}^{2}}{s}, \frac{m_{P}^{2}}{\mu^{2}}\right)=\ln \left(\frac{m_{P}^{2}}{\mu^{2}}\right)+\frac{8 m_{P}^{2}}{s}-\frac{5}{3}+\sigma_{P}^{3} \ln \left(\frac{\sigma_{P}+1}{\sigma_{P}-1}\right), \quad \sigma_{P}=\sqrt{1-4 m_{P}^{2} / s}, \\
\Gamma_{\rho}(s)=-\frac{m_{\rho} s}{96 \pi^{2} f_{\pi}^{2}} \operatorname{Im}\left[A\left(\frac{m_{\pi}^{2}}{s}, \frac{m_{\pi}^{2}}{m_{\rho}^{2}}\right)+\frac{1}{2} A\left(\frac{m_{K}^{2}}{s}, \frac{m_{K}^{2}}{m_{\rho}^{2}}\right)\right],
\end{gathered}
$$

where $f_{\pi}=92.42 \pm 0.07 \pm 0.25 \mathrm{MeV}$ [16], $m_{K}, m_{\rho}$ are the masses of $K^{ \pm}$and $\rho$ mesons. This pion form factor gives good description of the experimental data up to the energies of the order of $1 \mathrm{GeV}$ in terms of $m_{\pi}, m_{K}, m_{\rho}$ and $f_{\pi}$. To obtain the resonance contribution to $\sigma^{h}$ the Breit-Wigner representation has been exploited. The contribution of $\sigma_{\text {background }}^{h}$ was found by fitting of the experimental points [5, 6, 7, 8, 9, 10, 11, 12, 13. For description of the experimental data we have used polinomial fit: $R(s)=\sum_{k=0}^{n} c_{k}(\sqrt{s})^{k}$ of high order on $\mathrm{n}$. The coefficients $c_{k}$ were determined by means of the least-squares method. The region of $\sqrt{s}>60 \mathrm{GeV}$ was described by QCD formula, following from the assumption of the asymptotic freedom with six quarks. The results of our calculations are presented in the table. The total contribution of HVP to hyperfine muonium splitting is equal to:

$$
\Delta E_{h f s}^{h}(\mu e)=(3.5988 \pm 0.1045)\left(\frac{\alpha}{\pi}\right)^{2} \frac{m_{e} m_{\mu}}{m_{\pi}^{2}} \tilde{E}^{F}=0.2397 \pm 0.0070 k H z .
$$

This value is in accord with the result obtained in [3]: $\Delta E_{h f s}^{h}=0.250 \pm 0.016 \mathrm{kHz}$. The more than twice higher precision of (11) is explained by the account of new experimental data and by more accurate data treatment [11]. The new value (11) allows to increase the accuracy of testing radiative corrections to the ground state hyperfine splitting in 
muonium. It is important because a precision of hyperfine muonium splitting measurement in the new Los Alamos experiment is of order of $10^{-2} \mathrm{kHz}$. Much of theoretical efforts was spent in the last years in order to calculate new contributions to the muonium hyperfine splitting of a high degree in $\alpha$ and $m_{e} / m_{\mu}$ [17, 18, 19]. Theoretical value $\Delta E_{h f s}^{t h}=4463302.69$ (1.34) (0.21) (0.16) $\mathrm{kHz}$ [17], taking into account (11), is in good agreement with experimental result $\Delta E_{h f s}^{e x p}=4463302.88$ (16) kHz [1]]. The first and second errors in $\Delta E_{h f s}^{t h}$ reflect the uncertainties in the measurements of $\alpha^{-1}$ [20] and $m_{\mu} / m_{e}$. The third error is purely theoretical and dominated by the coefficients in the $\alpha(Z \alpha)^{2}$, $\alpha(Z \alpha)^{3} \ln (Z \alpha)$ corrections $[18$.

\begin{tabular}{|c|c|c|}
\hline final state & energy range $\sqrt{s}, \mathrm{GeV}$ & $\Delta E_{h f . s}^{h}(\mu e) /\left(\frac{\alpha}{\pi}\right)^{2} \frac{m_{e} m_{\mu}}{m_{\pi}^{2}} \tilde{E}^{F}$ \\
\hline$\rho, \omega \rightarrow 2 \pi$ & $(0.28,0.81)$ & $2.0518 \pm 0.0134$ \\
$\omega \rightarrow 3 \pi$ & $(0.42,0.81)$ & $0.1869 \pm 0.0061$ \\
$\phi$ & & $0.2122 \pm 0.0077$ \\
$J / \Psi$ & & $0.0538 \pm 0.0059$ \\
$\Upsilon$ & $(0.81,1.4)$ & $0.0006 \pm 0.0001$ \\
hadrons & $(1.4,2.2)$ & $0.5562 \pm 0.0260$ \\
hadrons & $(2.2,3.1)$ & $0.2406 \pm 0.0199$ \\
hadrons & $(3.1,5.0)$ & $0.1252 \pm 0.0110$ \\
hadrons & $(5.0,10.0)$ & $0.0906 \pm 0.0122$ \\
hadrons & $(10.0,40.0)$ & $0.0560 \pm 0.0015$ \\
hadrons & $(40.0,60.0)$ & $0.0227 \pm 0.0007$ \\
hadrons & $\sqrt{s} \geq 60.0$ & $0.0012 \pm 0.0001$ \\
hadrons & Summary contribution & 0.0010 \\
\hline \multicolumn{2}{|c}{} & \\
\hline
\end{tabular}

We are grateful to Prof. T. Kinoshita for suggestion to update the calculation of HVP contribution to muonium hyperfine splitting and to Prof. F. Jegerlehner for helpful discussions of experimental data. This work was supported by the Russian Foundation for Basic Research (grant no. 98-02-16185) and the Program "Universities of Russia" (grant 2759).

\section{References}

[1] Dvoeglazov V.V., Faustov R.N., Tukhtyaev Yu.N. //Particles and Nuclei. 1994. V.25. N1. P.144.

[2] Sapirstein J.R., et al. //Phys. Rev. 1984. D29. N10. P.2290.

[3] Karimkhodzaev A., Faustov R.N. //Yad. Fiz. 1991. V.53. P.1012.

[4] Martynenko A.P., Faustov R.N. //Theor. Math. Phys. 1985. V.64. P.765.

[5] Dolinsky S.I., et al. //Phys. Rep. 1991. V.C202. P.99.

[6] Bisello D., et al. //Phys. Let. 1989. V.B220. P.321. 
[7] Adachi I., et al. //Phys. Let. 1990. V.B234. P.525.

[8] Edwards C., et al. //SLAC-PUB-5160. 1990.

[9] Blinov A.E., et al. //Z. Phys. 1996. V.C70. P.31.

[10] Akhmetshin R.R., et al. //Phys. Let. 1995. V.B364. P.199.

[11] Eidelman S., Jegerlehner F. //Z.Phys. 1995. C67. P.585.

[12] Martinovic L., Dubnicka S. //Phys. Rev. 1992. D42. P.88

[13] Swartz M.L. //Phys. Rev. 1996. D53. P.5268.

[14] Martynenko A.P., Faustov R.N. //Phys. Atom. Nucl. 1998. V.61. P.471.

[15] Guerrero F., Pich A. //Phys. Let. 1997. V.B412. P.382.

[16] Review of Particle Properties //Phys. Rev. 1996. D54. N1. Part 1.

[17] Kinoshita T., Nio M. //Phys. Rev. 1996. V.D53. P.4909.

[18] Kinoshita T., Nio M. //Phys. Rev. 1997. V.D55. P.7267.

[19] Eides M.I., Grotch H., Shelyuto V.A. //Phys. Rev. 1998. V.D58. P.1308.

[20] Kinoshita T. //Rep. Prog. Phys. 1996. V.59. P.1459. 